OPEN ACCESS

Edited by:

James (Jim) David Cotter,

University of Otago, New Zealand

Reviewed by:

Joseph Isaac Shapiro,

Marshall University, USA

Lewis John James,

Loughborough University, UK

*Correspondence:

lan B. Stewart

i.stewart@qut.edu.au

Specialty section:

This article was submitted to

Exercise Physiology,

a section of the journal

Frontiers in Physiology

Received: 03 November 2016

Accepted: 13 January 2017

Published: 01 February 2017

Citation:

Stewart IB, Dias B, Borg DN, Bach AJE, Feigl B and Costello JT (2017) Intraocular Pressure Is a Poor Predictor of Hydration Status following Intermittent Exercise in the Heat. Front. Physiol. 8:36

doi: 10.3389/fphys.2017.00036

\section{Intraocular Pressure Is a Poor Predictor of Hydration Status following Intermittent Exercise in the Heat}

\author{
Ian B. Stewart ${ }^{1 *}$, Brittany Dias ${ }^{1}$, David N. Borg ${ }^{1}$, Aaron J. E. Bach ${ }^{1}$, Beatrix Feigl ${ }^{2}$ and \\ Joseph T. Costello ${ }^{1,3}$ \\ 1 School of Exercise and Nutrition Sciences and Institute of Health and Biomedical Innovation, Kelvin Grove, Queensland \\ University of Technology, Brisbane, QLD, Australia, ${ }^{2}$ School of Biomedical Sciences and Institute of Health and Biomedical \\ Innovation, Kelvin Grove, Queensland University of Technology, Brisbane, QLD, Australia, ${ }^{3}$ Extreme Environments Laboratory, \\ Department of Sport and Exercise Science, University of Portsmouth, Portsmouth, UK
}

Current hydration assessments involve biological fluids that are either compromised in dehydrated individuals or require laboratory equipment, making timely results unfeasible. The eye has been proposed as a potential site to provide a field-based hydration measure. The present study evaluated the efficacy and sensitivity of intraocular pressure (IOP) to assess hydration status. Twelve healthy males undertook two $150 \mathrm{~min}$ walking trials in $40^{\circ} \mathrm{C} 20 \%$ relative humidity. One trial matched fluid intake to body mass loss (control, CON) and the other had fluid restricted (dehydrated, DEH). IOP (rebound tonometry) and hydration status (nude body mass and serum osmolality) were determined every $30 \mathrm{~min}$. Body mass and serum osmolality were significantly $(p<0.05)$ different between trials at all-time points following baseline. Body mass losses reached $2.5 \pm 0.2 \%$ and serum osmolality $299 \pm 5 \mathrm{mOsmol} . \mathrm{kg}^{-1}$ in DEH. A significant trial by time interaction was observed for $\operatorname{IOP}(p=0.042)$, indicating that over the duration of the trials IOP declined to a greater extent in the DEH compared with the CON trial. Compared with baseline measurements IOP was reduced during DEH (150 min: $-2.7 \pm 1.9 \mathrm{~mm} \mathrm{Hg}$; $p<0.05$ ) but remained stable in CON (150 min: $-0.3 \pm 2.4 \mathrm{~mm} \mathrm{Hg}$ ). However, using an IOP value of $13.2 \mathrm{~mm} \mathrm{Hg}$ to predict a $2 \%$ body mass loss resulted in only $57 \%$ of the data being correctly classified (sensitivity $55 \%$ and specificity $57 \%$ ). The use of $\Delta$ IOP $(-2.4 \mathrm{~mm} \mathrm{Hg})$ marginally improved the predictive ability with $77 \%$ of the data correctly classified (sensitivity: 55\%; specificity: $81 \%$ ). The present study provides evidence that the large inter-individual variability in baseline IOP and in the IOP response to progressive dehydration, prevents the use of IOP as an acute single assessment marker of hydration status.

Keywords: hydration assessment, eye, intraocular pressure, dehydration, exercise, heat 


\section{INTRODUCTION}

Current best-practice human hydration assessments include osmolality of blood, saliva, or urine; specific gravity or color of urine; and changes in body mass compared to a baseline collected over several days (Armstrong, 2007; Cheuvront et al., 2010, 2013; Kenefick and Cheuvront, 2012). These procedures are either expensive, invasive, require clinical laboratory equipment, rely on a non-dehydrated baseline criterion or on body fluids that are compromised in a dehydrated individual. Reviews of hydration assessment techniques have highlighted the need to develop field indices that are suitable for the evaluation of large groups of people, involved in athletic or challenging occupational situations, where dynamic (involving a baseline criterion) measurements are not necessary (Armstrong, 2007).

Recently the eye has been identified (Sollanek et al., 2012; Sherwin et al., 2015) as having the potential to provide a valid hydration assessment in field settings, where the use of invasive procedures is limited. The relationship between ocular fluids (tear and aqueous humor), blood pressure and plasma osmolality has provided a case for tear fluid osmolarity (Fortes et al., 2011), tear break-up time (Sweeney et al., 2013), and intraocular pressure (IOP) (Hunt et al., 2012) as potential non-invasive measures of hydration status.

IOP is governed by the rates of formation and drainage of aqueous humor. Aqueous is continually being formed, filtering from the capillaries in the ciliary processes, flowing through the anterior chamber, and draining from the eye through the limbus and the scleral venous sinus. The production of aqueous humor is under tight neuro-endocrine regulation; with its flow through the anterior chamber influenced by hydrostatic, oncotic and osmotic pressures and its outflow regulated by the autonomic nervous system (Coca-Prados and Escribano, 2007).

Hyperosmolality of the blood caused by high intensity short duration exercise has been associated with reduced IOP (Marcus et al., 1970; Stewart et al., 1970). Several researchers have also suggested that low intensity long duration exercise in a hot environment resulting in sweating induced hypovolemia and subsequent hyperosmolality (as opposed to acidosis from high intensity exercise) could lower the rate of aqueous formation and consequently reduce IOP (Marcus et al., 1970; Harris et al., 1994). However, these studies did not require participants to exercise for a sufficient duration, or in a hot environment, to elicit a change in hydration status.

To date only two studies have assessed IOP over a prolonged duration and/or in a hot environment where an individual would experience significant body mass losses using different methods of IOP assessment. The first involved a $24 \mathrm{~h}$ march $\left(17-32^{\circ} \mathrm{C}\right.$, $45-85 \%$ relative humidity) where IOP progressively declined for the first $15 \mathrm{~h}$, at which time serum osmolality peaked (Ashkenazi et al., 1992). Forty-eight hours after completing the march, a reduction in IOP was observed, and again was accompanied by a rise in serum osmolality. At both time points a statistically significant moderate correlation $(r=-0.679$ and -0.649 , respectively, $p<0.001$ ) between IOP and serum osmolality was observed (Ashkenazi et al., 1992). More recently a small sample pilot study required participants to complete three $30 \mathrm{~min}$ walking bouts in a controlled environment $\left(43^{\circ} \mathrm{C}, 20 \%\right.$ relative humidity) (Hunt et al., 2012) and observed statistically significant moderate relationships between IOP and plasma osmolality $(r=-0.682)$, and change in body mass $(r=0.507)$.

Currently, the efficacy and sensitivity of IOP to determine changes in body mass associated with sweating induced hypovolemia have only been conducted in uncontrolled environments (Ashkenazi et al., 1992) or in a small pilot study (Hunt et al., 2012). Due to the potential feasibility of using IOP as a field based measure of hydration status in various sporting, occupational and clinical settings, the aim of the present investigation was to determine if IOP was associated with hydration status (body mass loss and serum osmolality) following exercise in the heat with and without fluid restriction. It was hypothesized that IOP would be reduced to a greater extent during exercise with fluid restriction, concomitant with modest hypohydration ( $>2 \%$ body mass loss) and increased serum osmolality.

\section{METHODS}

\section{Ethical Approval}

The testing protocols carried out in this study were approved by the Queensland University of Technology Human Research Ethics Committee. Participants were informed of the procedures and had any questions answered to their satisfaction prior to giving their oral and written consent to participate. The study conformed to the current Declaration of Helsinki guidelines.

\section{Participants}

Twelve healthy, physically active males (mean $\pm \mathrm{SD}$ ): age $24 \pm 2$ year, height $178 \pm 6 \mathrm{~cm}$, mass $75 \pm 7 \mathrm{~kg}, \dot{\mathrm{VO}}_{2 \max }$ $56 \pm 4 \mathrm{~mL} \cdot \mathrm{kg}^{-1} \cdot \mathrm{min}^{-1}$, sum of eight skinfolds $75 \pm 29 \mathrm{~mm}$ ) with normal ocular health as confirmed by an optometrist volunteered to participate. Exclusion criteria included any history of ocular disease involving raised eye pressure (or existing glaucoma or ocular hypertension).

\section{Experimental Design}

Participants were required to attend the laboratory on three occasions. The first laboratory visit involved eye testing, to determine high contrast visual acuity (Snellen chart) and health of the anterior and posterior eye (slit lamp biomicroscopy, funduscopy and IOP) by an experienced optometrist. The first visit also involved the determination of maximal aerobic power by an incremental treadmill running test to exhaustion and skin fold assessment of body composition, as previously described (Stewart et al., 2014). The remaining two trials, separated by a minimum of 7 days, involved five $30 \mathrm{~min}$ walking bouts. To control for the effects of circadian rhythm on IOP both walking trials commenced at the same time of day and differed only in the provision of fluid, with the participants either receiving no fluid throughout (to induce body mass losses, $\mathrm{DEH}$ ) or fluid replacement (with the aim to maintain body mass, CON). The order of the two walking trials was counterbalanced across participants. 


\section{Experimental Protocol}

The two walking trials followed a similar protocol. Participants were asked to avoid heavy exercise and the consumption of alcohol, caffeine and tobacco in the $24 \mathrm{~h}$ prior to each walking trial. To ensure euhydration, participants were instructed to consume $30 \mathrm{~mL} \cdot \mathrm{kg}^{-1}$ body mass of fluid (either water or sports drink) between 4 and $10 \mathrm{pm}$ the night before each session, and a further $250 \mathrm{~mL}$ of fluid the morning of the trial (at least $1 \mathrm{~h}$ prior to trial commencement). The participants were also given a calibrated (Hunt and Stewart, 2008) ingestible core temperature sensor (CorTemp, HQ Inc, Palmetto, FL, USA) to swallow the evening prior.

Upon arriving at the laboratory participants were asked to collect a mid-stream urine sample that was assessed for specific gravity (USG). Participants with a USG value $<1.020$ were classified as euhydrated (23 of 24 trials) and those with higher values ( 1 of 24 trials) were provided with an additional $500 \mathrm{~mL}$ of water to be consumed prior to the commencement of the walking trials. A chest strap (Polar Team2, Kempele, Finland) and data logger (CorTemp, HQ Inc, Palmetto, FL, USA) were then fitted to provide continuous heart rate and core temperature recordings, respectively.

Participants were then seated and a cannula was inserted in the left antecubital fossa to attain venous blood samples. Following at least $10 \mathrm{~min}$ of seated rest IOP and blood pressure from the right arm, using the auscultatory method, were obtained and blood samples drawn. Intraocular pressure was measured by an optometrist using a handheld contact (rebound) tonometer (TA01i, icare ${ }^{\circledR}$, Helsinki, Finland). The device measures the IOP in $<0.1 \mathrm{~s}$ and averages six readings to minimize deviation and to produce a calculated measurement value. The IOP measurement was performed in duplicate (triplicate if difference was $>1 \mathrm{~mm} \mathrm{Hg}$ ) for the right eye only (Fernandes et al., 2005). The closest two IOP values were used to obtain an average intraocular pressure for the participant for each time point. Blood samples were collected into $5 \mathrm{~mL}$ serum separating vacutainers for the determination of serum osmolality, $6 \mathrm{~mL}$ K3 EDTA vacutainers for the determination of hemoglobin concentration $(\mathrm{Hb})$, haematocrit (Hct) and blood lactate (Stewart et al., 2005). $\mathrm{Hb}$ and $\mathrm{Hct}$ were used to calculate the percent change in plasma volume (PV) during the trial (Dill and Costill, 1974). Nude body mass measurements were then obtained to the nearest $50 \mathrm{~g}$ (Tanita BWB-600, Wedderburn, Australia).

Participants then entered the environmental chamber $\left(40^{\circ} \mathrm{C}\right.$, $20 \%$ relative humidity, $4.7 \mathrm{~km} \cdot \mathrm{h}^{-1}$ air flow) and commenced walking at $5 \mathrm{~km} \cdot \mathrm{h}^{-1}$ and $1 \%$ gradient with core temperature and heart rate recorded and monitored continuously. Following 30 min the participants were removed from the environmental chamber into an air-conditioned laboratory and had $10 \mathrm{~min}$ of seated rest, after which IOP, blood pressure, blood collection, and nude body mass (after towel drying) were determined, in that order. This was repeated five times for a total of 150 min walking which equated to a total distance of $12.5 \mathrm{~km}$ for all participants.

During the fluid provision trial, $300 \mathrm{~mL}$ of room temperature $\left(\sim 22^{\circ} \mathrm{C}\right)$ water was provided in the first 30 min walking bout and in the remaining four walking bouts water provision was equated to the body mass loss in the preceding walking bout.
To ensure the fluid consumption had no subsequent effect on the measurement of IOP all fluid was consumed within the first 10 min of the walking bout (Brucculeri et al., 1999). Food, two biscuits and a banana, equating to a weight of $\sim 90 \mathrm{~g}$, was provided in both trials every hour.

\section{Statistical Analysis}

A power calculation using $\mathrm{G} *$ Power 3 software was performed in order to determine the required sample size for the experiment. Using an effect size from data previously collected in our laboratory (Cohen's $d=0.8, n=7$; Hunt, 2011), with $\alpha$ and power levels set at 0.05 and 0.8 , respectively, a sample of twelve participants was calculated to provide sufficient statistical power to detect changes in IOP during progressive dehydration.

The normal distribution of data was confirmed using descriptive methods (kurtosis, skewness, outliers and distribution plots) and inferential statistics (Shapiro-Wilk Test). Continuous variables were summarized as mean \pm standard deviation (unless otherwise stated). A two way repeated measures analysis of variance (ANOVA) was performed to assess the effects of time (baseline, 30, 60, 90, 120, and $150 \mathrm{~min}$ ) and trial (DEH and CON) on IOP, indicators of hydration status, heat strain, and blood pressure variables. Post-hoc analysis, using a Bonferroni correction, were conducted where appropriate. A Pearson's correlation coefficient was determined to observe the relationship between IOP and indicators of hydration status, heat strain, blood pressure and lactate across all trials and time points. Where a statistically significant relationship was observed, a univariate general linear model, with participant ID as a random effect, was utilized to determine statistical significance. This was to account for the within-participant correlation likely present within the data (due to repeated measures), and provides an average equation of the linear association from the association within each participant. Confidence intervals around the slope of the line were calculated using the $t$ statistic for eleven degrees of freedom. Finally, the sensitivity and specificity of IOP and $\triangle I O P$ to identify a $2 \%$ loss in body mass, in accordance with the ACSM Position Stand in Exercise and Fluid Replacement (Sawka et al., 2007) and other recent literature (Muñoz et al., 2013; Cheuvront and Kenefick, 2014) was determined. Statistical significance for all analysis was set at the $p<0.05$ level.

\section{RESULTS}

\section{Baseline Data}

IOP, body mass (CON $76.3 \pm 8.4$, DEH $76.2 \pm 8.7 \mathrm{~kg}$ ), serum osmolality, core temperature, heart rate, mean arterial pressure and blood lactate were similar $(p>0.05$; Table 1) at baseline before each trial.

\section{Dehydration Protocol}

All twelve participants completed the $150 \mathrm{~min}$ of exercise in the CON and DEH trials and no adverse events were recorded. DEH resulted in significant $(p<0.001)$ body mass losses and increases in serum osmolality compared with the CON trial (Table 1). Plasma volume was also significantly reduced in the $\mathrm{DEH}$ compared with the CON trial (DEH-CON: $-5.1 \pm 3.4 \%$, 
TABLE 1 | Physiological changes observed during the fluid restriction (DEH) and provision (CON) trials.

\begin{tabular}{|c|c|c|c|c|c|c|}
\hline & Baseline & $30 \mathrm{~min}$ & $60 \mathrm{~min}$ & $90 \mathrm{~min}$ & $120 \mathrm{~min}$ & $150 \mathrm{~min}$ \\
\hline \multicolumn{7}{|c|}{ IOP $(\mathrm{mm} \mathrm{Hg})$} \\
\hline CON & $14.4 \pm 4.1$ & $15.5 \pm 3.9$ & $14.7 \pm 3.9$ & $14.1 \pm 4.0$ & $14.5 \pm 3.5$ & $14.2 \pm 4.0$ \\
\hline \multicolumn{7}{|c|}{$\triangle$ BODY MASS (\%) } \\
\hline CON & & $0.0 \pm 0.1$ & $-0.1 \pm 0.1$ & $-0.1 \pm 0.1$ & $-0.1 \pm 0.2$ & $-0.2 \pm 0.2$ \\
\hline CON & $291 \pm 5$ & $291 \pm 3$ & $291 \pm 4$ & $291 \pm 3$ & $292 \pm 4$ & $292 \pm 3$ \\
\hline $\mathrm{DEH}$ & $292 \pm 3$ & $293 \pm 3^{*}$ & $294 \pm 3^{*}$ & $297 \pm 4^{*}$ & $298 \pm 4^{*}$ & $299 \pm 5^{*}$ \\
\hline \multicolumn{7}{|c|}{ CORE TEMPERATURE $\left({ }^{\circ} \mathrm{C}\right)$} \\
\hline CON & $37.2 \pm 0.3$ & $37.4 \pm 0.2$ & $37.5 \pm 0.2$ & $37.6 \pm 0.2$ & $37.6 \pm 0.2$ & $37.6 \pm 0.2$ \\
\hline $\mathrm{DEH}$ & $37.1 \pm 0.3$ & $37.4 \pm 0.2$ & $37.6 \pm 0.2$ & $37.7 \pm 0.2^{*}$ & $37.9 \pm 0.2^{\star}$ & $38.0 \pm 0.2^{*}$ \\
\hline \multicolumn{7}{|c|}{ MEAN ARTERIAL PRESSURE (mm Hg) } \\
\hline CON & $89 \pm 8$ & $88 \pm 8$ & $88 \pm 6$ & $88 \pm 5$ & $88 \pm 6$ & $89 \pm 6$ \\
\hline $\mathrm{DEH}$ & $90 \pm 6$ & $91 \pm 6$ & $91 \pm 8$ & $90 \pm 7$ & $91 \pm 7$ & $89 \pm 9$ \\
\hline \multicolumn{7}{|c|}{ BLOOD LACTATE (mmol.L $\left.{ }^{-1}\right)$} \\
\hline CON & $1.03 \pm 0.46$ & $0.98 \pm 0.44$ & $0.73 \pm 0.47$ & $0.93 \pm 0.54$ & $0.77 \pm 0.41$ & $0.94 \pm 0.56$ \\
\hline DEH & $1.31 \pm 0.74$ & $0.92 \pm 0.49$ & $0.89 \pm 0.57$ & $1.16 \pm 1.06$ & $1.09 \pm 0.85$ & $1.20 \pm 0.81$ \\
\hline
\end{tabular}

Data are mean $\pm S D(n=12)$. Significantly different to control at same time point * $(p<0.05) ;{ }^{\dagger}(p<0.001)$.

$p=0.001, n=10)$. No significant differences were observed in mean arterial pressure or blood lactate concentration, however heart rate and core temperature were significantly elevated $(p<0.05)$ in the DEH trial at the 120 and $150 \mathrm{~min}$ and 90, 120, and 150 min time points, respectively (Table $\mathbf{1}$ ).

\section{IOP}

The typical error of measurement for IOP, utilizing the baseline data from both trials, was calculated to be $1.65 \mathrm{~mm} \mathrm{Hg}$. No significant main effect for trial was observed (CON $14.6 \pm 3.7$, DEH $14.0 \pm 3.3 \mathrm{~mm} \mathrm{Hg}, p=0.257)$. A significant main effect for time $(p<0.001)$ and trial by time interaction was observed for IOP $(p=0.042$, Table 1$)$, indicating that over the duration of the trials IOP declined to a greater extent in the DEH compared with the CON trial. However, utilizing a Bonferroni correction for multiple comparisons, no single time-point maintained statistical significance. Similarly, when using the LSD post hoc analysis, no differences were observed.

Significant correlations $(p<0.05)$ were observed between IOP and body mass loss $(r=0.181)$, blood pressure $(r=0.501)$, and blood lactate $(r=0.190)$. As such these variables were entered into a univariate general linear model as covariates (individually) with IOP as a dependent variable and participant number as a random factor, to account for the repeated measurements. Only body mass loss was found to be significantly associated (Table 2).

When a body mass loss of 2\% (Sawka et al., 2007) was taken as a criterion limit for the presence of hypohydration using the regression equation, IOP was predicted to be $13.2 \mathrm{~mm} \mathrm{Hg}$. Figure 1A displays the relationship of IOP and body mass loss for each participant, with reference to these cut-off limits for hydration status. Of 120 data points (10 per participant), 43 were in a false positive region (IOP $<13.2 \mathrm{~mm} \mathrm{Hg}$, but body mass loss $<2 \%$ ), 57 were true negatives (IOP $>13.2 \mathrm{~mm} \mathrm{Hg}$ and body mass loss $<2 \%$ ), and 11 were true positive (IOP $<13.2 \mathrm{~mm} \mathrm{Hg}$ and body mass loss $>2 \%$ ). Overall $57 \%$ of the data were correctly classified by these limits, resulting in a test sensitivity of $55 \%$ and specificity of $57 \%$.

\section{$\Delta \mathrm{IOP}$}

Normalising the IOP to individual baseline values, $\triangle \mathrm{IOP}$ (Figure 2), produced significant main effects of trial (CON $0.14 \pm 1.9, \mathrm{DEH}-1.63 \pm 0.77 \mathrm{~mm} \mathrm{Hg}, p=0.002)$, time $(p<0.001)$ and their interaction $(p=0.020)$. Significant post-hoc comparisons, adjusted for multiple comparisons, were observed at 30, 90, 120, and $150 \mathrm{~min}$ (Figure 2).

$\Delta \mathrm{IOP}$ was significantly related to body mass loss $(r=0.526)$, serum osmolality $(r=-0.385)$ and core temperature $(r=-0.314)$. Univariate general linear model revealed a significant association for $\triangle \mathrm{IOP}$ with body mass loss, serum osmolality and core temperature (Table 2). At a $2 \%$ loss in body mass, $\triangle \mathrm{IOP}$ was predicted to be $-2.4 \mathrm{~mm} \mathrm{Hg}$. Utilizing this cut-off 19 data points were classified as false positives $(\Delta \mathrm{IOP}$ $<-2.4 \mathrm{~mm} \mathrm{Hg}$ and body mass loss $<2 \%$ ) and 9 false negatives $(\Delta \mathrm{IOP}<-2.4 \mathrm{~mm} \mathrm{Hg}$ and body mass loss $>2 \%)$. Eleven true positives and 81 true negatives were identified. Using $\Delta \mathrm{IOP} 77 \%$ of the data was correctly classified by these limits (sensitivity: 55\%; specificity: 81\%; Figure 1B). 
TABLE 2 | Univariate general linear model for IOP, $\Delta$ IOP, and covariates, with participant ID as a random factor.

\begin{tabular}{|c|c|c|c|c|c|c|c|}
\hline & $\mathbf{F}$ & Degrees of freedom & Significance & Intercept (SE) & Slope & 95\% Cl Low & 95\% Cl High \\
\hline \multicolumn{8}{|l|}{ ABSOLUTE IOP } \\
\hline$\Delta$ Body mass * & 22.096 & 1,107 & $<0.001$ & $14.75(1.164)$ & 0.77 & 0.41 & 1.13 \\
\hline Blood pressure & 0.552 & 1,131 & 0.459 & $4.91(3.92)$ & 0.03 & -0.05 & 0.01 \\
\hline Blood lactate & 0.004 & 1,129 & 0.952 & $14.29(1.01)$ & 0.02 & -0.66 & 0.70 \\
\hline \multicolumn{8}{|l|}{$\Delta$ IOP } \\
\hline$\Delta$ Body mass * & 56.352 & 1,107 & $<0.001$ & $0.26(0.88)$ & 1.33 & 0.94 & 1.72 \\
\hline Serum osmolality & 62.920 & 1,106 & $<0.001$ & $94(41)$ & -323 & -412 & -233 \\
\hline Core temperature & 22.976 & 1,101 & $<0.001$ & $127.78(1.58)$ & -3.42 & -4.99 & -1.85 \\
\hline
\end{tabular}

*Only data at 30, 60, 90, 120, and 150 min time points was used in this analysis as baseline values were "0" for all participants.

SE-standard error of intercept.

95\% Cl-95\% confidence interval around the slope of the line.
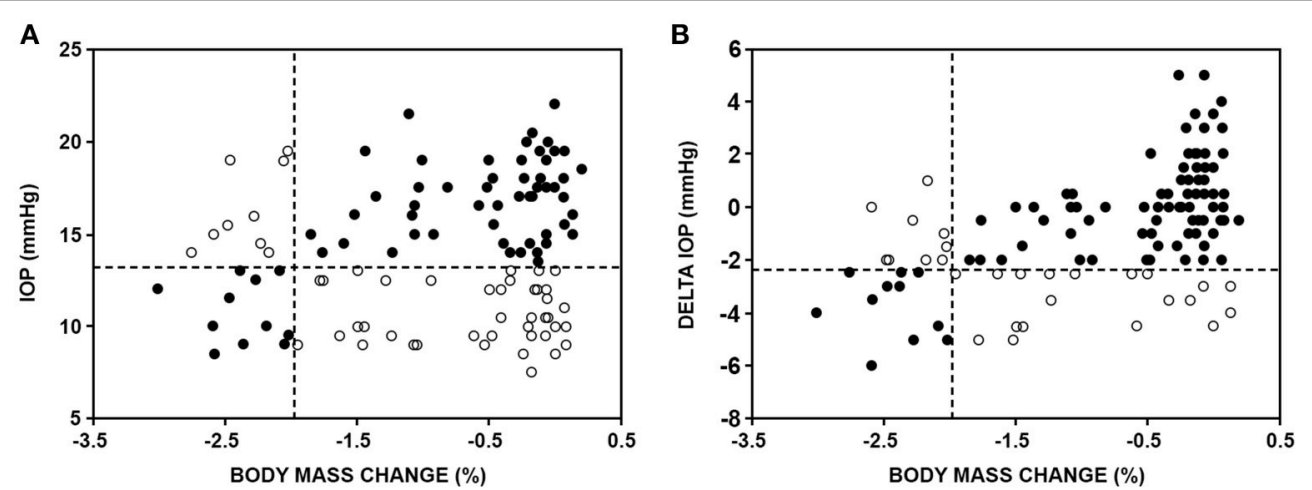

FIGURE 1 | (A) Sensitivity and specificity capability of IOP using a $13.2 \mathrm{~mm} \mathrm{Hg}$ criterion value to assess a $2 \%$ body mass loss. Dashed lines represent -2\% body mass change and $13.2 \mathrm{~mm} \mathrm{Hg}$ IOP. (B) Sensitivity and specificity capability of a $\Delta \mathrm{IOP}$ using a $-2.4 \mathrm{~mm}$ Hg criterion value to assess a $2 \%$ body mass loss. Dashed lines represent $-2 \%$ body mass change and $-2.4 \mathrm{~mm} \mathrm{Hg}$ IOP. Solid circles represent correct classification (true positive and negative) and open circles incorrect classification (false positive and negative).

\section{DISCUSSION}

This study is the first to experimentally evaluate the efficacy and sensitivity of using IOP to assess hydration status following intermittent exercise in the heat, with and without fluid restriction. Assessing thermal hypohydration using ocular fluids has recently gained interest in sports medicine literature (Fortes et al., 2011; Hunt et al., 2012; Sollanek et al., 2012; Sherwin et al., 2015) and IOP, in particular, may be appealing to sports medicine practitioners, clinicians, and researchers because the procedure is non-invasive, causes minimal discomfort, requires minimal training to perform accurately, and provides a reading within seconds. The novel findings of this investigation were: (1) in partial agreement with our initial hypothesis, a statistically significant interaction was observed between IOP and the level of hypohydration; however, there was no difference in IOP at any time during exercise in the heat irrespective of fluid provision or restriction (Table 1), and (2) using an IOP value of $13.2 \mathrm{~mm} \mathrm{Hg}$ as a criterion reference to assess a $2 \%$ loss in body mass resulted in only $57 \%$ of the data being correctly classified (Figure 1A). Thus, evidence from the present study does not support the use of IOP as an acute single assessment index of hypohydration.
In accordance with the experimental design, there was a systematic and significantly greater decline in body mass observed in the DEH compared to the CON trial (Table 1), averaging $0.5 \%$ per 30 min of treadmill walking. In conjunction with the body mass loss, serum osmolality also increased with progressive dehydration (Table 1) to values associated with a significant hypertonic-hypovolemia (Cheuvront et al., 2010). Hypohydration increases the heat strain experienced by those undertaking physical activity in the heat (Armstrong et al., 1997; Sawka et al., 2001), and previous studies that have induced body mass losses $>2 \%$ also routinely observed decrements in endurance physical performance (Sawka et al., 2007; Cheuvront and Kenefick, 2014). Therefore, the level of hypohydration observed in the fluid restriction trial of this study was of practical significance.

Fluctuations in IOP result from alterations in the rate of formation of the aqueous humor within the posterior chamber and/or the drainage of the aqueous humor from the anterior chamber of the eye. The rate of aqueous humor drainage is primarily influenced by anatomical structures and venous pressure (Brubaker, 1991) and has been reported to be uninfluenced by exercise (Stewart et al., 1970; Hong et al., 2014). 


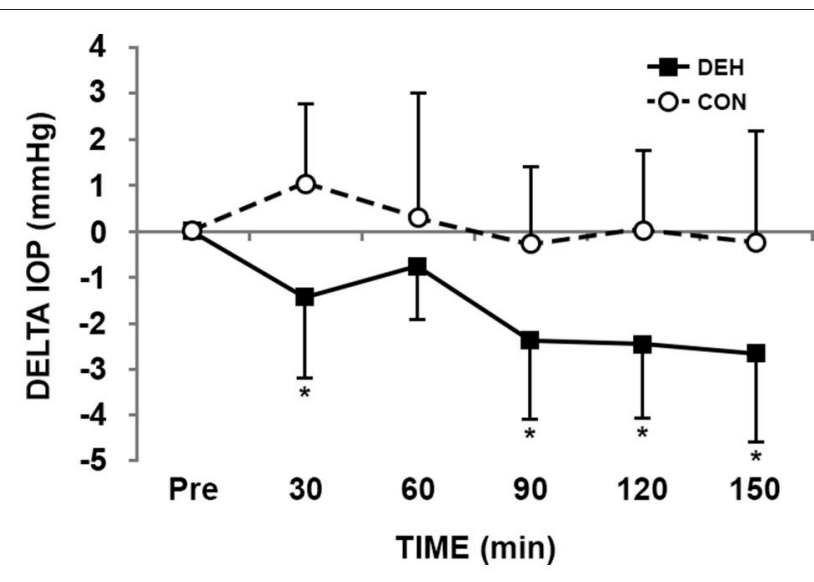

FIGURE 2 | $\triangle$ IOP from baseline in the fluid restriction (DEH) and provision (CON) trials. Significantly different to control at same time point ${ }^{*} p<0.05$.

Active transport, ultrafiltration, and diffusion are responsible for the formation of the aqueous humor (Brubaker, 1991). Of these diffusion is thought to be most important during fluid ingestion and/or exercise, as active transport and ultrafiltration have been shown to be uninvolved in acute changes of IOP in these situations (Brucculeri et al., 1999). Water is the main constituent of aqueous humor and it enters the posterior chamber by osmosis (Brubaker, 1991). Hyperosmotic agents (i.e., mannitol, glycerol, and isosorbide) have been shown to reduce IOP by creating a blood-ocular osmotic pressure gradient, thereby lowering the ocular tension via dehydration (Smith and Drance, 1962). Exercise-induced hypohydration also raises plasma osmolality, creating an osmotic gradient, favoring the movement of water from the aqueous humor to the blood. This would reduce the rate of aqueous humor formation and lower IOP (Ashkenazi et al., 1992; Risner et al., 2009). The current study provides empirical evidence to support this mechanism as a statistically significant relationship was found between serum osmolality and $\triangle \mathrm{IOP}$ (Table 2). The slope of the relationship was negative, indicating that IOP is reduced when serum osmolality is increased. Body mass loss was also significantly associated with both absolute IOP and $\triangle I O P$ (Table 2), further supporting the effects of hydration status. Although the CON trial isolated the effects of body water deficit by replicating the absolute exercise intensity, changes in body posture and diurnal effects, it should be noted that a causal relationship cannot be concluded from the associations observed in the current study.

Fluid ingestion has also been shown to influence IOP (Brucculeri et al., 1999; Read and Collins, 2010). Acute ingestion of $1 \mathrm{~L}$ of fluid has been documented to cause a $1-2 \mathrm{~mm} \mathrm{Hg}$ increase in IOP that peaks after 10-15 min and is still elevated at 30 min (Brucculeri et al., 1999; Read and Collins, 2010), but has returned to baseline at a time point between 30 and $45 \mathrm{~min}$ (Brucculeri et al., 1999). The increased IOP was postulated to be in response to gastric distension eliciting a sympathetic reflex increase in systemic arterial and vena caval pressure (Brucculeri et al., 1999). The increased vena caval pressure in turn would elevate episcleral venous pressure, minimizing aqueous drainage and subsequently elevating IOP. It is unlikely that the ingestion of water, independent of its influence on hydration status, influenced IOP in the current study as all measurements were recorded $>30 \mathrm{~min}$ after the fluid was consumed and the total volume of fluid consumed $(376 \pm 73 \mathrm{~mL})$ would have produced a significantly smaller degree of gastric distension. Further, given fluid ingestion, irrespective of absorption per se, can alter the fluid regulatory response (Figaro and Mack, 1997), additional research is warranted to examine the effect of using a dehydration model that also includes some fluid consumption.

IOP is also known to be reduced following exercise (Risner et al., 2009; Hong et al., 2014). The decline in IOP following short duration high intensity dynamic exercise coincides with the rise in blood lactate and plasma osmolality (Marcus et al., 1970; Stewart et al., 1970). In comparison, it has previously been demonstrated that short duration low intensity exercise produces a small decline in IOP, without these changes in blood lactate and plasma osmolality (Harris et al., 1994). These findings suggest an independent effect of exercise intensity. While blood lactate was significantly correlated with absolute IOP $(r=0.190)$, this relationship became insignificant when corrected for repeated measurements within each participant (Table 2). Similarly, there was no difference in blood lactate between the DEH and CON trials (Table 1). The absolute workload, of $5 \mathrm{~km} \cdot \mathrm{h}^{-1}$ and $1 \%$ grade represented a relative intensity for each participant of $20 \pm 6 \% \dot{\mathrm{VO}}_{2}$ max which was significantly lower than the previous study (Harris et al., 1994) that reported changes in IOP without differences in blood lactate or $\mathrm{pH}$. The absolute workload was also consistent between trials, yet we observed a significant difference in the IOP response to exercise-induced hypohydration (Figure 2). Therefore, it could be postulated that the IOP response occurred independently of aerobic exercise intensity, blood lactate or water consumption, supporting our primary hypothesis that IOP is reduced to a greater extent during exercise in the heat with fluid restriction, concomitant with modest hypohydration (2-3\% body mass loss) and increased serum osmolality.

Some thermoregulatory and cardiovascular variables differed between the $\mathrm{DEH}$ and CON trial and should be considered as potential factors influencing the IOP response. The present study observed a significantly elevated core temperature in the $\mathrm{DEH}$ trial compared to the CON trial from the 90 min time period to the end of the trial. The magnitude of this effect was on average $0.3^{\circ} \mathrm{C}$, range $0.1-0.8^{\circ} \mathrm{C}$ (Table 1 ). This elevation is a normal thermoregulatory response to exercise in the heat with fluid restriction; however, it does indicate a potential confounder to the above conclusion. It could be argued that the IOP response observed may be influenced by core temperature instead of hydration status per se, with a negative correlation observed with $\triangle \mathrm{IOP}(r=-0.314, p<0.001)$ but not between absolute IOP and core temperature $(r=-0.075, p=0.383)$ (Table 2). Heart rate was also increased from $120 \mathrm{~min}$ in the DEH trials compared to CON (Table 1). However, there was no significant relationship between absolute $(r=-0.003, p=0.976)$ or $\Delta \mathrm{IOP}$ $(r=-0.143, p=0.119)$ with heart rate. Our findings are supported by other researchers who have previously observed no 
relationship between heart rate and IOP (Ashkenazi et al., 1992; Karabatakis et al., 2004), but a negative association been $\Delta I O P$ and core temperature (Hunt et al., 2012).

Although the current data suggest an association between IOP and hydration status, there is limited potential for IOP to be used as a simple and practical technique to indicate hydration status in non-clinical settings (i.e., sporting or occupational environments). A body mass loss of $2 \%$ was chosen as a criterion level of hypohydration, as this level has previously been associated with decrements in physical endurance performance, increased heat strain, and increased risk of developing heat illness (Armstrong et al., 1997; Sawka et al., 2001; Cheuvront and Kenefick, 2014). Using the relationship between body mass loss and IOP, the corresponding IOP cut-off was predicted to be $13.2 \mathrm{~mm} \mathrm{Hg}$. The application of these cut-off limits to the IOP and body mass loss relationship can be observed in Figure 1A and highlight only $57 \%$ of the data was correctly classified with these limits. IOP at baseline ranged between 8.5 and $22 \mathrm{~mm} \mathrm{Hg}$, while in agreement with population norms (David et al., 1992) this does highlight a large degree of inter-individual variability. Three participants (25\%) had an IOP lower than the cut-off when adequately hydrated at baseline. Further as the trial progressed, all participants evidenced a decrease in IOP, however, the IOP of four participants (33\%) did not fall below the cut-off limit in spite of becoming dehydrated (evidenced by $>2.5 \%$ body mass loss). This suggests that the individual variability in IOP may be too large to establish a set limit value to indicate hypohydration without a euhydrated criterion baseline. Further, in comparison to other commonly used markers to diagnose exercise-induced hypohydration of $\geq 2 \%$ body mass loss (Muñoz et al., 2013), serum (sensitivity: $83 \%$, specificity: $82 \%$ ), saliva $(86,91 \%)$ and urine $(83,83 \%)$ osmolality, and urine volume $(79,79 \%)$ and specific gravity $(81,81 \%)$ all have been shown to have greater sensitivity and specificity compared to the IOP results presented within this study $(55,57 \%)$.

Despite the high individual variability in IOP a decline during the exercise-induced hypohydration was observed in all the participants. Therefore, we examined the use of a change score,

\section{REFERENCES}

Armstrong, L. (2007). Assessing hydration status: the elusive gold standard. J. Am. Coll. Nutr. 26, 575-584. doi: 10.1080/07315724.2007.10719661

Armstrong, L. E., Maresh, C. M., Gabaree, C. V., Hoffman, J. R., Kavouras, S. A, Kenefick, R. W., et al. (1997). Thermal and circulatory responses during exercise: effects of hypohydration, dehydration, and water intake. J. Appl. Physiol. (1985). 82, 2028-2035.

Ashkenazi, I., Melamed, S., and Blumenthal, M. (1992). The effect of continuous strenuous exercise on intraocular pressure. Invest. Ophthalmol. Vis. Sci. 33, 2874-2877.

Brubaker, R. F. (1991). Flow of aqueous humor in humans [The Friedenwald Lecture]. Invest. Ophthalmol. Vis. Sci. 32, 3145-3166.

Brucculeri, M., Hammel, T., Harris, A., Malinovsky, V., and Martin, B. (1999). Regulation of intraocular pressure after water drinking. J. Glaucoma 8, 111-116. doi: 10.1097/00061198-199904000-00005

Cheuvront, S. N., Ely, B. R., Kenefick, R. W., and Sawka, M. N. (2010). Biological variation and diagnostic accuracy of dehydration assessment markers. Am. J. Clin. Nutr. 92, 565-573. doi: 10.3945/ajcn.2010.29490 from baseline, as a potential indicator of a change in hydration status. Using the relationship between body mass loss and $\Delta \mathrm{IOP}$ from baseline, a $2 \%$ body mass loss corresponded to $\triangle \mathrm{IOP}$ of $-2.4 \mathrm{~mm} \mathrm{Hg}$ and slightly improved the classification accuracy to $77 \%$ (Figure 1B) and the test specificity (81\%), but not the sensitivity (55\%). The limited number of observations $>2 \%$ body mass loss (16\% of the data) in the current study significantly influences the IOP test sensitivity, regardless its diagnostic ability in the current study was only slightly better than random chance.

In conclusion, IOP is progressively reduced during exerciseinduced hypohydration, but remains stable if hydration is maintained during exercise in the heat. The present study provides novel evidence to suggest that IOP is significantly correlated to hydration status, likely due to the effect of a rise in serum osmolality on the rate of formation of aqueous humor. However, large inter-individual variability in baseline IOP and in the IOP response to progressive dehydration prevent IOP use, as measured by rebound tonometry, as an acute single assessment marker of hydration status.

\section{AUTHOR CONTRIBUTIONS}

IS and JC conceived and designed the research, analyzed the data and drafted the manuscript. $\mathrm{BD}, \mathrm{DB}$, and $\mathrm{AB}$ performed the experiments. IS, JC, and $\mathrm{BF}$ interpreted results of the experiments. All authors edited and revised the manuscript. All authors approved the final version of the manuscript and agree to be accountable for all aspects of the work in ensuring that questions related to the accuracy or integrity of any part of the work are appropriately investigated and resolved. All persons designated as authors qualify for authorship, and all those who qualify for authorship are listed.

\section{ACKNOWLEDGMENTS}

The authors would like to thank Ms Connie Wishart for assisting in the biochemical analysis.

Cheuvront, S. N., and Kenefick, R. W. (2014). Dehydration: physiology, assessment, and performance effects. Compr. Physiol. 4, 257-285. doi: 10.1002/cphy.c130017

Cheuvront, S. N., Kenefick, R. W., Charkoudian, N., and Sawka, M. N. (2013). Physiologic basis for understanding quantitative dehydration assessment. Am. J. Clin. Nutr. 97, 455-462. doi: 10.3945/ajcn.112.044172

Coca-Prados, M., and Escribano, J. (2007). New perspectives in aqueous humor secretion and in glaucoma: the ciliary body as a multifunctional neuroendocrine gland. Prog. Retin. Eye Res. 26, 239-262. doi: 10.1016/ j.preteyeres.2007.01.002

David, R., Zangwill, L., Briscoe, D., Dagan, M., Yagev, R., and Yassur, Y. (1992) Diurnal intraocular pressure variations: an analysis of 690 diurnal curves. $\mathrm{Br}$. J. Ophthalmol. 76, 280-283. doi: 10.1136/bjo.76.5.280

Dill, D. B., and Costill, D. L. (1974). Calculation of percentage changes in volumes of blood, plasma, and red cells in dehydration. J. Appl. Physiol. 37, 247-248.

Fernandes, P., Diaz-Rey, J. A., Queiros, A., Gonzalez-Meijome, J. M., and Jorge, J. (2005). Comparison of the ICare rebound tonometer with the Goldmann tonometer in a normal population. Ophthalmic Physiol. Opt. 25, 436-440. doi: $10.1111 /$ j.1475-1313.2005.00327.x 
Figaro, M. K., and Mack, G. W. (1997). Regulation of fluid intake in dehydrated humans: role of oropharyngeal stimulation. Am. J. Physiol. 272, R1740-R1746.

Fortes, M. B., Diment, B. C., Di Felice, U., Gunn, A. E., Kendall, J. L., Esmaeelpour, M., et al. (2011). Tear fluid osmolarity as a potential marker of hydration status. Med. Sci. Sports Exerc. 43, 1590-1597. doi: 10.1249/MSS.0b013e3182 $0 \mathrm{e} 7 \mathrm{cb} 6$

Harris, A., Malinovsky, V., and Martin, B. (1994). Correlates of acute exerciseinduced ocular hypotension. Invest. Ophthalmol. Vis. Sci. 35, 3852-3857.

Hong, J., Zhang, H., Kuo, D. S., Wang, H., Huo, Y., Yang, D., et al. (2014). The short-term effects of exercise on intraocular pressure, choroidal thickness and axial length. PLoS ONE 9:e104294. doi: 10.1371/journal.pone.0104294

Hunt, A. P., Feigl, B., and Stewart, I. B. (2012). The intraocular pressure response to dehydration: a pilot study. Eur. J. Appl. Physiol. 112, 1963-1966. doi: 10.1007/s00421-011-2143-5

Hunt, A. P. (2011). Heat Strain, Hydration Status, and Symptoms of Heat Illness in Surface Mine Workers. Ph.D. dissertation, Queensland University of Technology, Brisbane, 248.

Hunt, A. P., and Stewart, I. B. (2008). Calibration of an ingestible temperature sensor. Physiol. Meas. 29, N71-N78. doi: 10.1088/0967-3334/29/11/N01

Karabatakis, V. E., Natsis, K. I., Chatzibalis, T. E., Lake, S. L., Bisbas, I. T., Kallinderis, K. A., et al. (2004). Correlating intraocular pressure, blood pressure, and heart rate changes after jogging. Eur. J. Ophthalmol. 14, 117-122.

Kenefick, R. W., and Cheuvront, S. N. (2012). Hydration for recreational sport and physical activity. Nutr. Rev. 70(Suppl. 2), S137-S142. doi: 10.1111/j.1753-4887.2012.00523.x

Marcus, D. F., Krupin, T., Podos, S. M., and Becker, B. (1970). The effect of exercise on intraocular pressure: i. human beings. Invest. Ophthalmol. Vis. Sci. 9, 749-752.

Muñoz, C. X., Johnson, E. C., Demartini, J. K., Huggins, R. A., McKenzie, A. L., Casa, D. J., et al. (2013). Assessment of hydration biomarkers including salivary osmolality during passive and active dehydration. Eur. J. Clin. Nutr. 67, 1257-1263. doi: 10.1038/ejcn.2013.195

Read, S. A., and Collins, M. J. (2010). Water drinking influences eye length and IOP in young healthy subjects. Exp. Eye Res. 91, 180-185. doi: 10.1016/j.exer.2010.04.015

Risner, D., Ehrlich, R., Kheradiya, N. S., Siesky, B., McCranor, L., and Harris, A. (2009). Effects of exercise on intraocular pressure and ocular blood flow: a review. J. Glaucoma 18, 429-436. doi: 10.1097/IJG.0b013e31818fa5f3
Sawka, M. N., Montain, S. J., and Latzka, W. A. (2001). Hydration effects on thermoregulation and performance in the heat. Comp. Biochem. Physiol. Mol. Integr. Physiol. 128, 679-690. doi: 10.1016/S1095-6433(01)00274-4

Sawka, M. N., Burke, L. M., Eichner, E. R., Maughan, R. J., Montain, S. J., and Stachenfeld, N. S. (2007). ACSM position stand: exercise and fluid replacement. Med. Sci. Sports Exerc. 39, 377-390. doi: 10.1249/mss.0b013e31802ca597

Sherwin, J. C., Kokavec, J., and Thornton, S. N. (2015). Hydration, fluid regulation and the eye: in health and disease. Clin. Exp. Ophthalmol. 43, 749-764. doi: 10.1111/ceo.12546

Smith, E. W., and Drance, S. M. (1962). Reduction of human intraocular pressure with intravenous mannitol. Arch. Ophthalmol. 68, 734-737. doi: 10.1001/archopht.1962.00960030738007

Sollanek, K. J., Kenefick, R. W., Walsh, N. P., Fortes, M. B., Esmaeelpour, M., and Cheuvront, S. N. (2012). Assessment of thermal dehydration using the human eye: what is the potential? J. Therm. Biol. 37, 111-117. doi: 10.1016/j.jtherbio.2011.12.006

Stewart, I. B., Stewart, K. L., Worringham, C. J., and Costello, J. T. (2014). Physiological tolerance times while wearing explosive ordnance disposal protective clothing in simulated environmental extremes. PLOS ONE 9:e83740. doi: 10.1371/journal.pone.0083740

Stewart, I. B., Bulmer, A. C., Sharman, J. E., and Ridgway, L. (2005). Arterial oxygen desaturation kinetics during apnea. Med. Sci. Sports Exerc. 37, 1871-1876. doi: 10.1249/01.mss.0000176305.51360.7e

Stewart, R. H., LeBlanc, R., and Becker, B. (1970). Effects of exercise on aqueous dynamics. Am. J. Ophthalmol. 69, 245-248. doi: 10.1016/0002-9394(70)91285-7

Sweeney, D. F., Millar, T. J., and Raju, S. R. (2013). Tear film stability: a review. Exp. Eye Res. 117, 28-38. doi: 10.1016/j.exer.2013.08.010

Conflict of Interest Statement: The authors declare that the research was conducted in the absence of any commercial or financial relationships that could be construed as a potential conflict of interest.

Copyright (c) 2017 Stewart, Dias, Borg, Bach, Feigl and Costello. This is an openaccess article distributed under the terms of the Creative Commons Attribution License (CC BY). The use, distribution or reproduction in other forums is permitted, provided the original author(s) or licensor are credited and that the original publication in this journal is cited, in accordance with accepted academic practice. No use, distribution or reproduction is permitted which does not comply with these terms. 